\title{
Escala diagramática para avaliação da severidade da mancha branca em milho
}

\author{
Paula Jimena Diniz Sachs ${ }^{1}$, Carmen Silvia Vieira Janeiro Neves², Marcelo Giovanetti Canteri² ${ }^{2}$ Luís Guilherme Sachs ${ }^{3}$
}

${ }^{1}$ Programa de pós-graduação em Agronomia da Universidade Estadual de Londrina-UEL -Londrina - PR- www.uel.br - pjdsachs@yahoo.com.br; ${ }^{2}$ Dep. Agronomia da Universidade Estadual de Londrina-UEL - Cx. Postal 6001 - CEP 86051-990 - Rodovia Celso Garcia Cid | Pr 445 Km 380 | Campus Universitário Londrina - PR - www.uel.br - Bolsista CNPq - csvjneve@uel.br; canteri@uel.br; ${ }^{3}$ Dep. Biologia e Tecnologia das Faculdades Luiz Meneghel/Universidade Estadual do Norte do Paraná -FALM /UENP - Br 369 km 54, CEP 86360-000 - Bandeirantes - PR www.ffalm.br - sachs@ffalm.br

Autor para correspondência: Marcelo Giovanetti Canteri (canteri@uel.br)

Data de chegada: 26/11/2007. Aceito para publicação em: 10/10/2011.

\section{RESUMO}

Sachs, P.J.D.; Neves, C.C.S.V.J.; Canteri, M.G.; Sachs, L.G. Escala diagramática para avaliação da severidade da mancha branca em milho. Summa Phytopathologica, v.37, n.4, p.202-204, 2011.

Escalas diagramáticas são importantes ferramentas para padronizar as estimativas da severidade de doenças em plantas. O objetivo deste trabalho foi desenvolver e validar uma escala diagramática para avaliação da severidade da mancha branca ou mancha de Phaeosphaeria em folhas de milho. A escala proposta ilustra os níveis de $1 ; 3 ; 6 ; 13$; $25 ; 43 ; 63$ e $79 \%$ de severidade no terço médio de imagens de folhas de milho. A validação da escala foi realizada com o auxílio de dez avaliadores (cinco sem experiência e cinco com experiência), os quais estimaram a severidade da doença em 50 folhas de milho com sintomas da mancha branca, com e sem a utilização da escala. Análises de regressão foram utilizadas para avaliar a precisão e acurácia. A avaliação da acurácia indicou que sem o uso da escala sete avaliadores apresentaram desvios sistemáticos nas estimativas, enquanto que com o uso da escala foram apenas dois avaliadores. A precisão representada pelo coeficiente de determinação $\left(\mathrm{r}^{2}\right)$ das regressões, variou de 0,86 a 0,95 para os avaliadores inexperientes sem escala e 0,91 a 0,98 com escala. Os avaliadores experientes apresentaram $\mathrm{r}^{2}$ variando de 0,91 a 0,95 sem escala e 0,94 e 0,98 com escala. A escala diagramática proposta melhorou a precisão e a acurácia das estimativas, independente da experiência do avaliador.

Palavras-chave adicionais: Phaeosphaeria maydis, estímulo visual, Webber-Fechner, Zea mays, feosféria.

\section{ABSTRACT}

Sachs, P.J.D.; Neves, C.C.S.V.J.; Canteri, M.G.; Sachs, L.G. Diagrammatic scale for assesment of the phaeosphaeria leaf spot severity in maize. Summa Phytopathologica, v.37, n.4, p.202-204, 2011.

The Diagrammatic scales are important tools to standardize the severity assessments of plant diseases. The objective of this work was to develop and to validate a diagrammatic scale for severity assessment of leaves spot Phaeosphaeria on maize. The proposed scale had the levels of $1 ; 3 ; 6 ; 13,25 ; 43 ; 63$ and $79 \%$ of severity in the third medium part of the maize leaf. Scale validation was performed by ten raters (five inexperienced and five experienced), who estimated the severity disease in 50 maize leafs showing Phaeosphaeria leaf spot symptoms, with and without the use of the scale. Analyses of regression was used to evaluate the precision and accuracy of the estimates. The evaluation of the accuracy indicated that without the use of the scale seven raters had presented systematic shunting lines in the estimates, whereas with the use of the scale they was only two raters. The precision represented for coefficient of determination $\left(\mathrm{r}^{2}\right)$ of the regressions, varied of 0.86 the 0.95 for the inexperienced raters without scale and 0.91 the 0.98 with scale. The proposal diagrammatic scale improved the precision and accuracy of the estimates, independent of the experience of the raters.

Keywords: Phaeosphaeria maydis, visual stimulus, Webber-Fechner, Zea mays.

A mancha branca do milho, também chamada de mancha foliar de Phaeosphaeria é uma doença de distribuição generalizada em áreas produtoras de milho no Brasil. Os primeiros trabalhos apontam o fungo Phaeosphaeria maydis como agente causal (2). Entretanto, trabalhos recentes sugerem que, ao menos no Brasil, a mancha é causada por um complexo microbiano, uma vez que mais de um patógeno pode causar sintomas semelhantes. Entre estes encontram-se a bactéria Pantoea ananas e os fungos Phyllosticta sp., Phoma sorghina e Sporormmiella sp. (9). De acordo com Paccola-Meirelles et al. (8) a bactéria Pantoea ananas é um dos principais agentes etiológicos envolvidos no estágio inicial da doença, sendo os fungos de ocorrência e infecção secundária.

Em estudos epidemiológicos e na avaliação da eficiência de medidas de controle há necessidade de métodos confiáveis, precisos e que apresentem resultados reprodutíveis na quantificação de doenças em plantas (1). Tais medidas são essenciais na quantificação da doença no espaço e no tempo (10). O método de avaliação varia com o agente etiológico e com a característica espacial da doença (3). No caso de doenças foliares, a severidade é a variável mais utilizada, sendo que sua avaliação é, normalmente, feita de forma subjetiva por meio de 
análises visuais e, por conseguinte, as escalas diagramáticas tornam-se ferramentas fundamentais em tais estudos (4).

Os objetivos do presente trabalho foram a elaboração e a validação de uma escala diagramática para avaliação da severidade da mancha branca em milho.

Em uma área com milho híbrido Dow 2B150, em estágio de grão farináceo, foram coletadas aleatoriamente 100 folhas, com diferentes níveis de severidade da mancha branca. O terço médio de cada folha foi escaneado individualmente utilizando-se escâner (Plustec OpticPro P12) em imagens com resolução de 300 dpi. Em seguida, cada folha foi analisada em computador quanto à proporção de área lesionada, utilizando-se o programa APS Assess, software para a quantificação de doenças em plantas através da análise de imagens digitais (6), obtendo-se assim a severidade real da doença em termos percentuais.

Para o desenvolvimento da escala determinou-se o limite mínimo (menor quantidade de doença observada visualmente) e máximo (quantidade de doença em que ocorre senescência). Os níveis intermediários de severidade foram determinados de acordo com a lei de Weber-Fechner de acuidade visual (6). A partir dos valores obtidos e respeitando a forma, distribuição e frequência das lesões, estabeleceuse uma escala diagramática com 8 níveis de severidade.

A validação da escala foi realizada através de avaliações de severidade feitas por dez pessoas, sendo cinco experientes e cinco inexperientes. Cada avaliador analisou 50 imagens de segmentos de folhas com diferentes níveis de severidade da doença em duas diferentes sessões, sem auxílio e com auxílio da escala diagramática. Foi determinado o desempenho dos avaliadores quanto à precisão, acurácia e variância dos erros absolutos. A precisão e a exatidão (acurácia) das estimativas visuais de cada avaliador foram determinadas por meio de análise de regressão linear, considerando a severidade real como variável independente e a severidade estimada pelo avaliador como variável dependente. A precisão das estimativas foi avaliada pelo coeficiente de determinação da regressão linear simples $\left(r^{2}\right)$ e pela variância dos erros absolutos (severidade estimada menos severidade real). A acurácia de cada avaliador foi determinada por meio do teste $t$ aplicado ao coeficiente angular da reta $(a)$ e ao coeficiente linear da reta $(b)$, ambos obtidos pela regressão linear (severidade real x severidade estimada) (7).

A escala diagramática elaborada apresentou oito níveis de severidade e teve como limite inferior e superior os valores de $1 \%$ e $79 \%$ de área lesionada, respectivamente. Os níveis intermediários, seguindo-se a lei de Weber-Fechner, foram ajustados para 3, 6, 13, 25, 43 e $63 \%$ de severidade (Figura 1). Valores de severidade acima de $79 \%$ não foram incluídos na escala, pois estes não foram observados a campo.

Na validação da escala diagramática, sem a sua utilização, os valores do intercepto relacionados aos avaliadores $\mathrm{C}, \mathrm{D}, \mathrm{E}$ e $\mathrm{H}$ diferiram significativamente de zero $(\mathrm{p}<0,01)$, indicando a presença de desvios constantes (Tabela 1). Esses avaliadores superestimaram consistentemente $(\mathrm{p}<0,01)$ a severidade da mancha de Phaeosphaeria, indicando a presença de desvios positivos constantes para todos os níveis de severidade da doença. Com a utilização da escala, os valores do intercepto dos avaliadores C e E diferiram significativamente de zero $(p<0,01)$, todos com desvios positivos constantes. Entretanto ocorreu decréscimo na superestimativa desses avaliadores (Tabela 1). Folhas onde severidades semelhantes são representadas por diferentes números e conseqüentemente tamanhos de lesões, freqüentemente geram diferentes estimativas, e a tendência é para superestimativa, quando o número de lesões é alto e seu tamanho pequeno (4). Em relação aos valores do coeficiente angular da reta, a maioria dos avaliadores (sete) apresentou valores significativamente diferentes de $1(\mathrm{p}<0,01)$ sem a utilização da escala diagramática, indicando a presença de desvios sistemáticos (Tabela 1). Com o uso da escala, somente os avaliadores Ae $\mathrm{J}$ apresentaram coeficientes angulares significativamente diferentes de $1(\mathrm{p}<0,01)$ (Tabela 1).

A precisão foi alta com e sem o uso da escala diagramática para ambos, avaliadores experientes e inexperientes (Tabela 1). O valor de $\mathrm{r}^{2}$ dos avaliadores inexperientes variou de 0,86 a 0,95 sem escala e

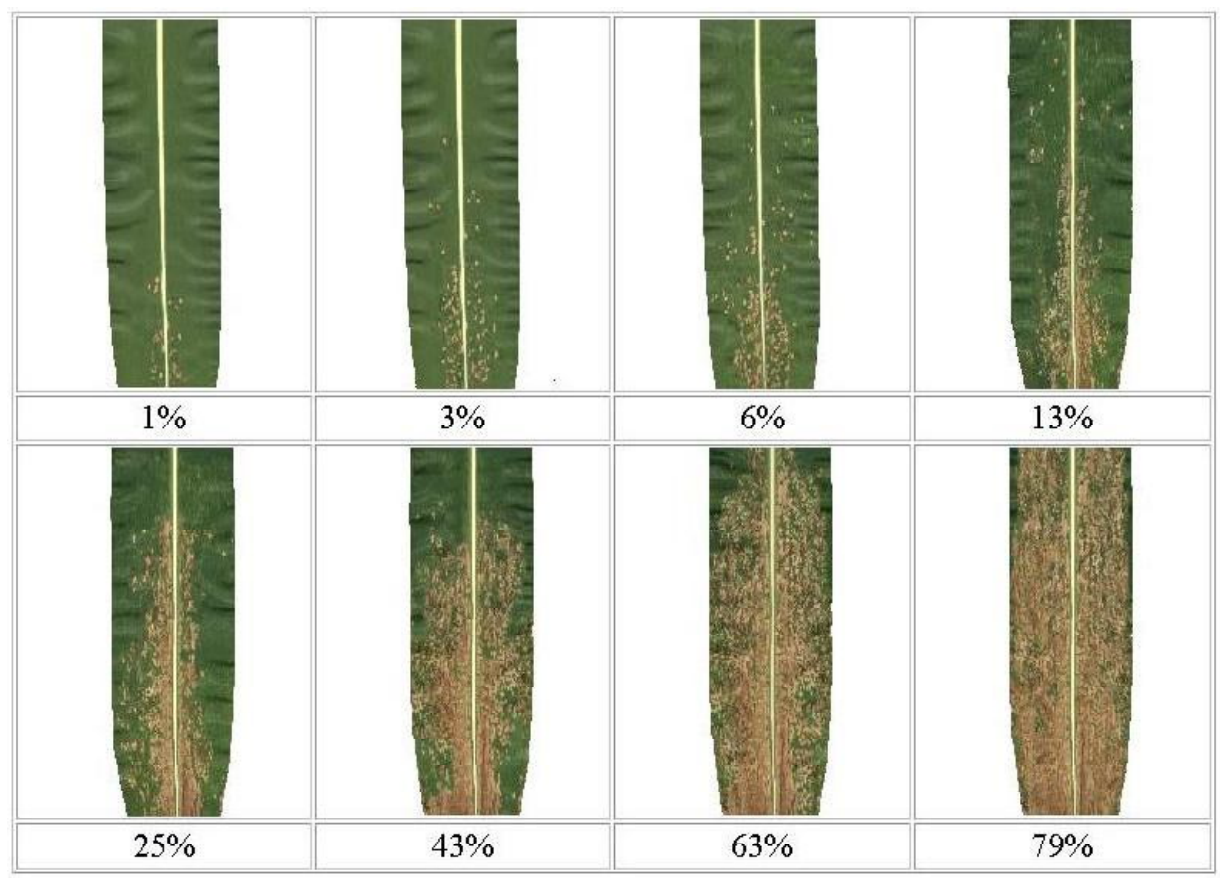

Figura 1. Escala diagramática para avaliação da severidade da mancha branca ou mancha foliar de Phaeosphaeria em milho. Percentagem da área foliar lesionada. 
Tabela 1. Intercepto (a), coeficiente angular da reta (b) e coeficiente de determinação $\left(\mathrm{r}^{2}\right)$ de equações de regressão linear simples para a severidade estimada da mancha branca em milho com e sem auxílio de escala diagramática para cada avaliador e para dados em conjunto (Regr. Geral).

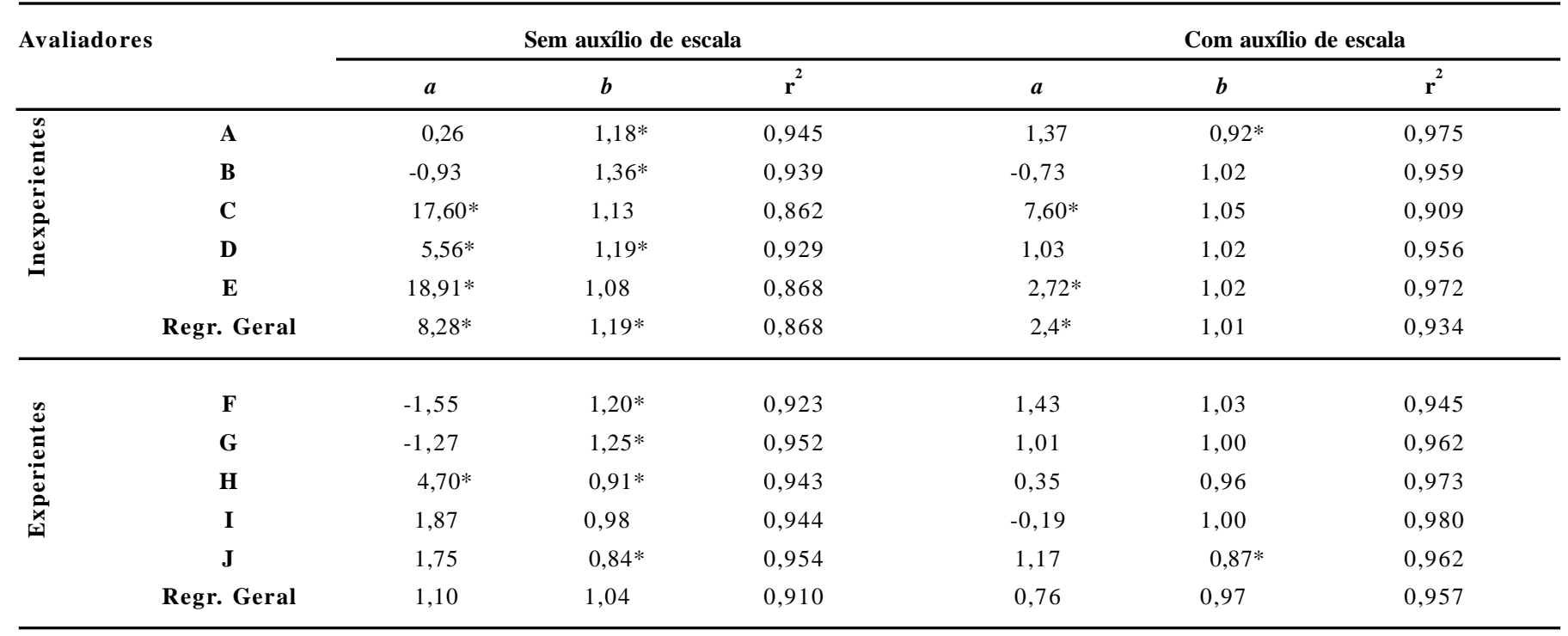

*situações nas quais as hipóteses $a=0$ e $b=1$, foram rejeitadas pelo teste $\mathrm{t}(\mathrm{p}<0,01)$.

0,91 a 0,98 com escala, ao passo que para os avaliadores experientes o $r^{2}$ variou de 0,91 a 0,95 sem escala e de 0,94 a 0,98 com escala. Para as avaliações visuais da mancha branca, realizadas com o auxílio da escala diagramática, a maioria dos avaliadores obtiveram valores $\left(\mathrm{r}^{2}\right)$ de precisão superiores a 95\% considerados desejáveis por Kranz (5). Os resultados deste estudo permitem concluir que o uso da escala diagramática proposta para quantificação da severidade da mancha branca em milho melhora a precisão e acurácia das estimativas, independente da experiência do avaliador.

\section{AGRADECIMENTOS}

Os autores expressam seus agradecimentos a todos os avaliadores que participaram das etapas de validação da escala diagramática.

\section{REFERÊNCIAS BIBLIOGRÁFICAS}

1. Bergamin Filho, A.; Amorim, L. Doenças de plantas tropicais: epidemiologia e controle econômico. São Paulo: Ed. Agronômica Ceres, 1996. 289 p.

2. Fantin, G.M.; Balmer, E. Método de inoculação e evolução dos sintomas da mancha foliar de Phaeosphaeria maydis em milho. Summa Phytopathologica, Botucatu, v.23, n.1, p.64-65, 1997.
3. Gaunt, R.E. The relationship between plant disease and severity and yield. Annual Review of Phytopathology, Palo Alto, v.33, n. 1, p.119-144, 1995.

4. Godoy, C.V.; Carneiro, S.M.T.P.; Iamauti, M.T.; Pria, M.D.; Amorim, L.; Berger, R.D.; Bergamin Filho, A. Diagrammatic scales for bean diseases: Development and validation. Journal of Plant Disease and Protection, Stuttgart, v.104, n.4, p.336-345, 1997.

5. Kranz, J. Measuring Plant Disease. In: Kranz, J.; Rotem, J. (Eds.) Experimental Techniques in Plant Disease Epidemiology. Berlim: Springer Verlag. 1988. p.35-50.

6. Lamari, L. Assess: Image analysis software for plant disease quantification. St. Paul: APS Press, 2002. 1CD-ROM.

7. Martins, M.C.; Guerzoni, R.A.; Câmara, G.M.S.; Mattiazzi, P.; Lourenço, S.A.; Amorim, L. Escala diagramática para a quantificação do complexo de doenças foliares de final de ciclo em soja. Fitopatologia Brasileira, Brasília, v.29, n.2, p.179-184, 2004.

8. Paccola-Meirelles, L.D.; Ferreira, A.S.; Meirelles, W.F.; Marriel, I.E.; Casela, C.R. Detection of a bacterium associated with a leaf spot disease of maize in Brazil. Journal of Phytopathology, Berlin, v.149, n.5, p.275-279, 2001.

9. Pereira, O.A. P; Carvalho, R.V.; Camargo, L.E.A. Doenças do milho (Zea mays L. ). In: Kimati, H.; Amorim, L.; Rezende, J.A.M.; Bergamin Filho, A.; Camargo, L.E.A. (Eds). Manual de Fitopatologia: Doenças das plantas cultivadas. 4. ed. São Paulo: Agronômica Ceres, 2005. v.2, p.477-488.

10. Zadoks, J.C. Methodology of epidemiological research. Annual Review of Phytopathology, Palo Alto, v.10, p.253-276, 1972. 